\title{
Editorial for the special issue: Health Management
}

\author{
George Dimas
}

Published online: 24 December 2010

(C) Springer-Verlag 2010

Health systems sector confronts major challenges worldwide that arise mainly from its dynamic and rapidly transformable environment.

Global characteristics like the current economic crisis, the evolution of new technologies and IT together with demography changes, create new facts which are added to the already existing concerns for the management of health systems.

Over the years extensive research from different disciplines has been conducted to issues related to health systems and solutions have been proposed that improve the performance of the systems. O.R. techniques saw sustained growth overtime in providing useful information for the efficient and effective management of health systems.

This issue of the journal is dedicated to Health Management and it was arranged on the grounds of the 21st National Congress of the Greek OR Society entitled "Decision Making in Health Systems" held in Athens on May 2009.

The special issue contains five papers selected from several submissions. Each submitted paper was peer-reviewed by at least two reviewers. The papers addressed are different.

The paper by Vozikis et al. applies the theory of Partially Observable Markov Decision Processes to patients with Ischemic Heart Disease (IHD) and proposes a method to accelerate value iteration to the therapy of IHD. The results show great promise as an alternative means for determining cost-effective monitoring and treatment policies to patients with IHD.

The next paper addresses the issue of productivity in public hospitals. Dimas et al. evaluate the productive performance of a sample of 22 Greek public hospitals over a 3-year period. Malmquist indices and Data Envelopment Analysis are employed to measure and decompose productivity while Tobit multivariate

G. Dimas $(\bowtie)$

Healthcare Management Department, Technological Educational Institute of Athens,

Agiou Spyridonos, 12210 Aigaleo Athens, Greece

e-mail: gdimas@teiath.gr 
analysis is used to determine whether environmental factors affect the hospital's productivity. The results suggest that productivity changes were dominated by the technical change component while hospital's inefficiency was attributed to an excessive increase of their expenditures.

The paper by Koulouriotis and Mantas analyses and compares applied forecasting techniques based on computational intelligence. It employs neural networks and the neural fuzzy system to forecast sales of a major Greek medical supplier. The results reveal that neural networks models can be a feasible solution and should be considered as a first class technique in the field of medical inventory.

The next two papers address issues related to simulation. The paper by Jahangirian et al. compares the results of literature reviews over the period 1997-2006 on the application of simulation in the sectors of Commerce, Defense and Healthcare. The purpose of the reviews is to see whether it is possible to learn any lessons from the first two sectors to be applied in Healthcare. Results provide clear evidence that Healthcare lags behind the other two sectors in appreciation of the role of simulation and particularly in terms of stakeholder engagement and consequently, in terms of implementation of simulation outcomes. Vlachos et al. use a Monte Carlo simulation approach to estimate the required inventory level for Umbilical Cord Blood (UCB) in Greece, an effective source of hematopoietic stem cells with increasing usage worldwide and to ensure an adequate probability that a patient finds a Human Leukocyte Antigen (HLA) compatible unit/donor. The results reveal that a 10,000 bank capacity ensures a $96 \%$ probability that a Greek transplant candidate finds a 4/6 HLA-matching unit.

We would like to thank all authors that contributed for the publication of this special issue. We are also grateful to reviewers for their quality reports that allowed us to reassure the high standards of papers accepted in this special issue and finally we sincerely thank the editor-in-chief for his continuous support to this effort. 